\title{
Global continuum and multiple positive solutions to one-dimensional $p$-Laplacian boundary value problem
}

Liping Wei ${ }^{1}$ and Ruyun $\mathrm{Ma}^{1 *}$

"Correspondence:

mary@nwnu.edu.cn

${ }^{1}$ Department of Mathematics,

Northwest Normal University,

Lanzhou, P.R. China

\section{Springer}

\begin{abstract}
We show the global structure of the set of positive solutions of a discrete Dirichlet problem involving the $p$-Laplacian difference operator suggesting suitable conditions on the weight function and nonlinearity. We obtain existence and multiplicity of positive solutions for $\lambda$ lying in various intervals in $\mathbb{R}$ by using the directions of a bifurcation and the Picone-type identity for discrete $p$-Laplacian operators.
\end{abstract}

MSC: 39A10; 39A12; 34G20

Keywords: Difference equation; $p$-Laplacian; Positive solutions; Bifurcation; Picone-type identity

\section{Introduction and main result}

Let $T>1$ be an integer, $\mathbb{T}:=[1, T]_{\mathbb{Z}}=\{1,2, \ldots, T\}, \hat{\mathbb{T}}:=\{0,1, \ldots, T+1\}$. In this paper, we are concerned with existence and multiplicity of positive solutions of the discrete boundary value problem

$$
\left\{\begin{array}{l}
\Delta\left[\varphi_{p}(\Delta u(x-1))\right]+\lambda h(x) f(u(x))=0, \quad x \in \mathbb{T}, \\
u(0)=u(T+1)=0,
\end{array}\right.
$$

where $\varphi_{p}(s)=|s|^{p-2} s, p>1, \lambda>0$ is the parameter, $f \in C\left(\mathbb{R}^{+}, \mathbb{R}^{+}\right), f(s)>0$ for all $s>0$ and $h: \hat{\mathbb{T}} \rightarrow \mathbb{R}^{+}$with $0<h_{*} \leq h(t) \leq h^{*}$ on $\mathbb{T}$ for some $h_{*}, h^{*} \in(0, \infty)$.

Existence of positive solutions for discrete boundary value problems involving the $p$ Laplacian difference operator has been studied by several authors, we refer to Agarwal et al. [1], Chu and Jiang [4], and [7, 8, 10, 12] as well as the references therein. Very recently, Nastasi et al. $[14,15]$ also obtained some existence results for discrete $(p, q)$-Laplacian equations. In particular, by virtue of bifurcation techniques, Bai and Chen [3] established some results of existence of positive solutions for (1.1) according to the asymptotic behavior of $f$ at 0 and $\infty$. However, the sublinear and superlinear conditions imposed on the nonlinearities only deduced a relatively simple "shape of the component", and they provided no information on the existence of at least three positive solutions.

(c) The Author(s) 2020. This article is licensed under a Creative Commons Attribution 4.0 International License, which permits use, sharing, adaptation, distribution and reproduction in any medium or format, as long as you give appropriate credit to the original author(s) and the source, provide a link to the Creative Commons licence, and indicate if changes were made. The images or other third party material in this article are included in the article's Creative Commons licence, unless indicated otherwise in a credit line to the material. If material is not included in the article's Creative Commons licence and your intended use is not permitted by statutory regulation or exceeds the permitted use, you will need to obtain permission directly from the copyright holder. To view a copy of this licence, visit http://creativecommons.org/licenses/by/4.0/. 
It is the purpose of this paper to show that (1.1) has three positive solutions for $\lambda$ lying in various intervals in $\mathbb{R}$ suggesting suitable conditions on the weight function and nonlinearity by using the directions of a bifurcation and the Picone-type identity (for related results, we refer to $[6,19,22])$ for discrete $p$-Laplacian operators due to Řehák [20]. We shall make the following assumptions:

(A1) $h: \hat{\mathbb{T}} \rightarrow \mathbb{R}^{+}$with $0<h_{*} \leq h(t) \leq h^{*}$ on $\mathbb{T}$ for some $h_{*}, h^{*} \in(0, \infty)$.

(A2) There exist $\alpha>0, f_{0}>0$, and $f_{1}>0$ such that

$$
\lim _{s \rightarrow 0^{+}} \frac{f(s)-f_{0} s^{p-1}}{s^{p-1+\alpha}}=-f_{1} .
$$

(A3) $f \in C\left(\mathbb{R}^{+}, \mathbb{R}^{+}\right), f(s)>0$ for all $s>0$ and $f_{\infty}:=\lim _{s \rightarrow \infty} \frac{f(s)}{s^{p-1}}=0$.

In assumption (A4) below and throughout, we use the following standard notations. Let $Y=\{u \mid u: \mathbb{T} \rightarrow \mathbb{R}\}$ with the norm $\|u\|_{Y}=\max _{t \in \mathbb{T}}|u(t)|$. Let $X=\{u: \hat{\mathbb{T}} \rightarrow \mathbb{R} \mid u(0)=$ $u(T+1)=0\}$ with the norm $\|u\|=\max _{t \in \hat{\mathbb{T}}}|u(t)|$.

Let $\mu_{1}$ be the first eigenvalue of the following problem:

$$
\left\{\begin{array}{l}
\Delta\left[\varphi_{p}(\Delta u(x-1))\right]+\mu h(x) \varphi_{p}(u(x))=0, \quad x \in \mathbb{T} \\
u(0)=u(T+1)=0
\end{array}\right.
$$

Then the first eigenvalue $\mu_{1}$ is the minimum of the Reyleigh quotient, that is,

$$
\mu_{1}=\inf \left\{\frac{\sum_{t=0}^{T}|\Delta u(t)|^{p}}{\sum_{t=0}^{T} h(t)|u(t)|^{p}}, u \in X\right\} .
$$

Let $\chi_{1}$ be the principal eigenvalue of the eigenvalue problem

$$
\left\{\begin{array}{l}
\Delta\left[\varphi_{p}(\Delta w(x-1))\right]+\chi \varphi_{p}(w(x))=0, \quad x \in[1, \hat{t}-1]_{\mathbb{Z}} \\
w(0)=0, w(\hat{t})=0
\end{array}\right.
$$

where $\hat{t} \in \mathbb{T}$ satisfies $\frac{T}{2} \leq \hat{t} \leq \frac{T+1}{2}$, and let $w_{1}$ be an eigenfunction corresponding to $\chi_{1}$.

Furthermore, we assume that

(A4) there exist $s_{0}>0$ and $0<\sigma<1$ such that

$$
\min _{s \in\left[s_{0}, \frac{1}{\sigma} s_{0}\right]} \frac{f(s)}{s} \geq \frac{f_{0}}{\mu_{1} h_{*}} \chi_{1}
$$

Arguing the shape of bifurcation, we have the following main result.

Theorem 1.1 Assume that (A1), (A2), (A3), and (A4) hold. Then there exist $\lambda_{*} \in\left(0, \mu_{1} / f_{0}\right)$ and $\lambda^{*}>\mu_{1} / f_{0}$ such that

(i) (1.1) has at least one positive solution if $\lambda=\lambda_{*}$;

(ii) (1.1) has at least two positive solutions if $\lambda_{*}<\lambda \leq \mu_{1} / f_{0}$;

(iii) (1.1) has at least three positive solutions if $\mu_{1} / f_{0}<\lambda<\lambda^{*}$;

(iv) (1.1) has at least two positive solutions if $\lambda=\lambda^{*}$;

(v) (1.1) has at least one positive solution if $\lambda>\lambda^{*}$. 
Remark 1.2 To obtain our main goals, we shall employ a bifurcation technique due to Sim and Tanaka [21]. They showed that one-dimensional $p$-Laplacian of differential equation coupled with Dirichlet boundary condition has three positive solutions. Moreover, several papers have also been devoted to elliptic equations involving the fractions of the Laplacian $($ see $[2,13])$. For other multiplicity results for related problems, we refer to [16-18].

The rest of the paper is organized as follows. In Sect. 2, we show the existence of bifurcation from the first eigenvalue for the corresponding problem according to the standard argument and the rightward direction of bifurcation. In Sect. 3, the change of direction of bifurcation is given. The final section is devoted to showing a priori bound of solutions for (1.1) and completing the proof of Theorem 1.1.

\section{Rightward bifurcation}

In this section, we study global bifurcation phenomena from the trivial branch with the rightward direction under suitable assumptions on $h$ and $f$. We need the following preliminary lemma.

Lemma 2.1 Assume that (A1) holds. Then the principal eigenvalue $\mu_{1}$ of (1.2) satisfies

(i) the eigenfunction $\phi_{1}$ corresponding to $\mu_{1}$ is of one sign in $\mathbb{T}$;

(ii) the algebraic multiplicity of $\mu_{1}$ is 1.

Proof (i) Let $\phi_{1}$ be the eigenfunction of (1.2) with $\mu_{1}$, then

$$
\left\{\begin{array}{l}
\Delta\left[\varphi_{p}\left(\Delta \phi_{1}(x-1)\right)\right]+\mu_{1} h(x) \varphi_{p}\left(\phi_{1}(x)\right)=0, \quad x \in \mathbb{T} \\
\phi_{1}(0)=\phi_{1}(T+1)=0
\end{array}\right.
$$

Multiplying the equation of (2.1) by $-\phi_{1}^{-}$and by a direct computation, one has

$$
-\sum_{t=0}^{T} \varphi_{p}\left(\Delta \phi_{1}(t)\right) \Delta \phi_{1}^{-}(t)=-\sum_{t=1}^{T} \mu_{1} h(t) \varphi_{p}\left(\phi_{1}(t)\right) \phi_{1}^{-}(t)=\sum_{t=1}^{T} \mu_{1} h(t)\left|\phi_{1}^{-}(t)\right|^{p} .
$$

Since

$$
\sum_{t=0}^{T}\left|\Delta \phi_{1}^{-}\right|^{p} \leq-\sum_{t=0}^{T} \varphi_{p}\left(\Delta \phi_{1}(t)\right) \Delta \phi_{1}^{-}(t)
$$

we obtain

$$
0<\sum_{t=0}^{T}\left|\Delta \phi_{1}^{-}\right|^{p} \leq \sum_{t=1}^{T} \mu_{1} h(t)\left|\phi_{1}^{-}(t)\right|^{p}
$$

According to the definition of $\mu_{1}$, we know that $\phi_{1}^{-}$is an eigenfunction of (1.2) with eigenvalue $\mu_{1}$.

We claim that $\phi_{1}^{-}>0$. Assume that there exists $t_{0} \in \mathbb{T}$ such that $\phi_{1}^{-}\left(t_{0}\right)=0$, then

$$
-\Delta\left[\varphi_{p}\left(\Delta \phi_{1}^{-}\left(t_{0}-1\right)\right)\right]=0,
$$


that is, $\varphi_{p}\left(\Delta \phi_{1}^{-}\left(t_{0}-1\right)\right)=\varphi_{p}\left(\Delta \phi_{1}^{-}\left(t_{0}\right)\right)=0$, which means

$$
\phi_{1}^{-}\left(t_{0}-1\right)=\phi_{1}^{-}\left(t_{0}+1\right)=0 .
$$

It deduces that $\phi_{1}^{-} \equiv 0$ from repeating the steps as above, which is a contradiction.

Consequently, $\phi_{1}=-\phi_{1}^{-}<0$ is of one sign in $\mathbb{T}$.

(ii) Let $u$ and $v$ be two eigenfunctions corresponding to $\mu_{1}$, we only need to prove that there exists $c \in \mathbb{R}$ such that $u=c v$.

From (i), we know that $u$ and $v$ are of one sign, we can suppose that $u>0, v>0$. Let $c=\min _{t \in \mathbb{T}} \frac{u(t)}{v(t)}$, then there exists $t_{0} \in \mathbb{T}$ such that $c=\frac{u\left(t_{0}\right)}{v\left(t_{0}\right)}$. Thus

$$
\varphi_{p}\left(u\left(t_{0}\right)\right)=\varphi_{p}\left(c v\left(t_{0}\right)\right) .
$$

From the equation of (1.2), one has

$$
\varphi_{p}\left(\Delta u\left(t_{0}-1\right)\right)-\varphi_{p}\left(\Delta u\left(t_{0}\right)\right)=\varphi_{p}\left(\Delta\left(c v\left(t_{0}-1\right)\right)\right)-\varphi_{p}\left(\Delta\left(c v\left(t_{0}\right)\right)\right) .
$$

Since

$$
\begin{aligned}
\varphi_{p}\left(\Delta u\left(t_{0}\right)\right)-\varphi_{p}\left(\Delta\left(c v\left(t_{0}\right)\right)\right) & =\varphi_{p}\left(u\left(t_{0}+1\right)-u\left(t_{0}\right)\right)-\varphi_{p}\left(c v\left(t_{0}+1\right)-c v\left(t_{0}\right)\right) \\
& =\varphi_{p}\left(u\left(t_{0}+1\right)-c v\left(t_{0}\right)\right)-\varphi_{p}\left(c v\left(t_{0}+1\right)-c v\left(t_{0}\right)\right)
\end{aligned}
$$

and $c=\min _{t \in \mathbb{T}} \frac{u(t)}{v(t)}$, one has $u\left(t_{0}+1\right) \geq c v\left(t_{0}+1\right)$, thus

$$
\varphi_{p}\left(\Delta u\left(t_{0}\right)\right)-\varphi_{p}\left(\Delta\left(c v\left(t_{0}\right)\right)\right) \geq 0 .
$$

By similar methods, we get

$$
\varphi_{p}\left(\Delta u\left(t_{0}-1\right)\right)-\varphi_{p}\left(\Delta\left(c v\left(t_{0}-1\right)\right)\right) \leq 0 .
$$

And accordingly,

$$
0 \leq \varphi_{p}\left(\Delta u\left(t_{0}\right)\right)-\varphi_{p}\left(\Delta\left(c v\left(t_{0}\right)\right)\right)=\varphi_{p}\left(\Delta u\left(t_{0}-1\right)\right)-\varphi_{p}\left(\Delta\left(c v\left(t_{0}-1\right)\right)\right) \leq 0 .
$$

It deduces that $u=c v$ from repeating the steps as above.

Moreover, coupled with (i) and (ii), the eigenfunction corresponding to $\mu_{1}$ can be chosen to be positive on $\mathbb{T}$.

Following similar arguments as in Lemma 2.2 of Bai and Chen [3], we have

Lemma 2.2 Assume that (A1), (A2), and (A3) hold. Then there exists an unbounded subcontinuum $\mathcal{C}$, which is emanating from $\left(\mu_{1} / f_{0}, 0\right)$ for $(1.1)$. Moreover, if $(\lambda, u) \in \mathcal{C}$, then $u$ is a positive solution of (1.1).

Lemma 2.3 Assume that (A1), (A2), and (A3) hold. Let $u$ be a positive solution of (1.1). Then there exists a constant $C$ independent of $u$ such that

$$
|\Delta u(x)| \leq \lambda^{\frac{1}{p-1}} C\|u\|, \quad x \in[0, T]_{\mathbb{Z}}
$$


Proof According to discrete Rolle's theorem (see [9]), there exists $x_{0} \in \mathbb{T}$ such that $\Delta u\left(x_{0}\right)=0$ or $\Delta u\left(x_{0}-1\right) \Delta u\left(x_{0}\right)<0$. Then, by a direct computation, it is easy to see that

$$
\varphi_{p}[\Delta u(x)]=\lambda \sum_{s=x+1}^{x_{0}} h(s) f(u(s)), \quad x \in \mathbb{T} .
$$

By virtue of (A2) and (A3), we get

$$
f(s) \leq f^{*} s^{p-1}, \quad s \geq 0
$$

for some $f^{*}>0$, it follows from (2.3) that

(i) if $\Delta u\left(x_{0}\right)=0$, then

$$
|\Delta u(x)|^{p-1}=\lambda \sum_{s=x+1}^{x_{0}} h(s) f(u(s)) \leq \lambda f^{*}\|u\|^{p-1} \sum_{s=x+1}^{x_{0}} h(s) \leq \lambda f^{*} \sum_{s=0}^{T+1} h(s)\|u\|^{p-1}
$$

(ii) if $\Delta u\left(x_{0}-1\right) \Delta u\left(x_{0}\right)<0$, then

$$
|\Delta u(x)|^{p-1}=\left.|| \Delta u\left(x_{0}\right)\right|^{p-2} \Delta u\left(x_{0}\right)+\lambda \sum_{s=x+1}^{x_{0}} h(s) f(u(s)) \mid \leq \lambda f^{*} \sum_{s=0}^{T+1} h(s)\|u\|^{p-1} .
$$

Lemma 2.4 Assume that (A1), (A2), and (A3) hold. Let $\left\{\left(\lambda_{n}, u_{n}\right)\right\}$ be a sequence of positive solutions to (1.1) which satisfies $\left\|u_{n}\right\| \rightarrow 0$ and $\lambda_{n} \rightarrow \mu_{1} / f_{0}$. Let $\phi_{1}(x)$ be an eigenfunction of (1.2) corresponding to $\mu_{1}$ which satisfies $\left\|\phi_{1}\right\|=1$. Then there exists a subsequence of $\left\{u_{n}\right\}$, again denoted by $\left\{u_{n}\right\}$, such that $u_{n} /\left\|u_{n}\right\|$ converges uniformly to $\phi_{1}$ on $\mathbb{T}$.

Proof Set $v_{n}:=u_{n} /\left\|u_{n}\right\|$. Then it is easy to see that $\left\|v_{n}\right\|=1$. It follows from Lemma 2.3 that $\left\|\Delta v_{n}\right\|$ is bounded, so there is a subsequence of $v_{n}$ uniformly convergent to a limit $v$. Furthermore, there exists a subsequence of it such that $\Delta v_{n}(0)$ converges to some constant $c$. We again denote by $\left\{v_{n}\right\}$ the subsequence. We note that $v \in Y, v(0)=v(T+1)=0$, and $\|v\|=1$. Rewriting the equation of (1.1) with $(\lambda, u)=\left(\lambda_{n}, u_{n}\right)$, we obtain

$$
\varphi_{p}\left(\Delta u_{n}(x)\right)=\varphi_{p}\left(\Delta u_{n}(0)\right)-\lambda_{n} \sum_{t=1}^{x} h(t) f\left(u_{n}(t)\right) .
$$

Dividing both sides of (2.5) by $\left\|u_{n}\right\|^{p-1}$, we get

$$
\varphi_{p}\left(\Delta v_{n}(x)\right)=\varphi_{p}\left(\Delta v_{n}(0)\right)-\lambda_{n} \sum_{t=1}^{x} h(t) \frac{f\left(u_{n}(t)\right)}{\varphi_{p}\left(u_{n}(t)\right)} \varphi_{p}\left(v_{n}(t)\right)=: w_{n}(x) .
$$

Since $u_{n}(x) \rightarrow 0$ for all $x \in \hat{\mathbb{T}}$, we can get $\frac{f\left(u_{n}(t)\right)}{\varphi_{p}\left(u_{n}(t)\right)} \rightarrow f_{0}$ for each fixed $t \in \hat{\mathbb{T}}$. It follows that $w_{n}(x)$ converges to

$$
w(x):=\varphi_{p}(c)-\mu_{1} \sum_{t=1}^{x} h(t) \varphi_{p}(v(t))
$$


for each fixed $x \in \hat{\mathbb{T}}$. Therefore, by recalling (2.6), one has

$$
v_{n}(x)=\sum_{s=0}^{x-1} \varphi_{p}^{-1}\left(w_{n}(s)\right)
$$

The fact coupled with (2.7) yields that $v_{n}(x)$ converges to

$$
v(x)=\sum_{s=0}^{x-1} \varphi_{p}^{-1}(w(s))=\sum_{s=0}^{x-1} \varphi_{p}^{-1}\left(\varphi_{p}(c)-\mu_{1} \sum_{t=1}^{s} h(t) \varphi_{p}(v(t))\right),
$$

which implies that $v$ is a nontrivial solution of (1.2) with $\lambda=\mu_{1}$, and hence $v \equiv \phi_{1}$.

Lemma 2.5 Assume that (A1), (A2), and (A3) hold. Let $\mathcal{C}$ be as in Lemma 2.2. Then there exists $\delta>0$ such that, for each $(\lambda, u) \in \mathcal{C}$ and $\left|\lambda-\mu_{1} / f_{0}\right|+\|u\| \leq \delta$, one has $\lambda>\mu_{1} / f_{0}$.

Proof Suppose on the contrary that there exists a sequence $\left\{\left(\lambda_{n}, u_{n}\right)\right\}$ such that $\left(\lambda_{n}, u_{n}\right) \in \mathcal{C}$, which satisfies $\lambda_{n} \rightarrow \mu_{1} / f_{0},\left\|u_{n}\right\| \rightarrow 0$, and $\lambda_{n} \leq \mu_{1} / f_{0}$. According to Lemma 2.4, there exists a subsequence of $\left\{u_{n}\right\}$, for convenience denoted by $\left\{u_{n}\right\}$, such that $\frac{u_{n}}{\left\|u_{n}\right\|}$ converges uniformly to $\phi_{1}$ on $\hat{\mathbb{T}}$, where $\phi_{1}(x)>0$ is the first eigenfunction of (1.2) with $\left\|\phi_{1}\right\|=1$. Multiplying the equation of $(1.1)$ with $(\lambda, u)=\left(\lambda_{n}, u_{n}\right)$ by $u_{n}$ and by a direct computation, one has

$$
\lambda_{n} \sum_{x=0}^{T} h(x) f\left(u_{n}(x)\right) u_{n}(x)=\sum_{x=0}^{T}\left|\Delta u_{n}(x)\right|^{p},
$$

and accordingly

$$
\lambda_{n} \sum_{x=0}^{T+1} h(x) f\left(u_{n}(x)\right) u_{n}(x) \geq \mu_{1} \sum_{x=0}^{T+1} h(x)|u(x)|^{p} .
$$

It follows from Lemma 2.4 that, after taking a subsequence and relabeling if necessary, $\frac{u_{n}}{\left\|u_{n}\right\|}$ converges to $\phi_{1}$ in $Y$.

$$
\sum_{x=0}^{T}\left|\Delta \phi_{1}(x)\right|^{p}=\mu_{1} \sum_{x=0}^{T} h(x)\left|\phi_{1}(x)\right|^{p}
$$

then together with (2.8) one has

$$
\lambda_{n} \sum_{x=0}^{T} h(x) f\left(u_{n}(x)\right) u_{n}(x)=\mu_{1} \sum_{x=0}^{T} h(x)\left|u_{n}(x)\right|^{p}-\zeta(n)\left\|u_{n}\right\|^{p}
$$

with a function $\zeta: \mathbb{N} \rightarrow \mathbb{R}$ satisfying

$$
\lim _{n \rightarrow \infty} \zeta(n)=0
$$


That is,

$$
\begin{aligned}
& \sum_{x=0}^{T+1} h(x) \frac{f\left(u_{n}(x)\right)-f_{0}\left[u_{n}(x)\right]^{p-1}}{\left[u_{n}(x)\right]^{p-1+\alpha}}\left|\frac{u_{n}(x)}{\left\|u_{n}\right\|}\right|^{p+\alpha} \\
& \quad=\frac{1}{\lambda_{n}\left\|u_{n}\right\|^{\alpha}}\left[\left(\mu_{1}-f_{0} \lambda_{n}\right) \sum_{x=0}^{T+1} h(x)\left|\frac{u_{n}(x)}{\left\|u_{n}\right\|}\right|^{p}-\zeta(n)\right] .
\end{aligned}
$$

From condition (A2), we have

$$
\sum_{x=0}^{T+1} h(x) \frac{f\left(u_{n}(x)\right)-f_{0}\left[u_{n}(x)\right]^{p-1}}{\left[u_{n}(x)\right]^{p-1+\alpha}}\left|\frac{u_{n}(x)}{\left\|u_{n}\right\|}\right|^{p+\alpha} \rightarrow-f_{1} \sum_{x=0}^{T+1} h(x)\left|\phi_{1}(x)\right|^{p+\alpha}<0
$$

and

$$
\sum_{x=0}^{T+1} h(x)\left|\frac{u(x)}{\left\|u_{n}\right\|}\right|^{p} \rightarrow \sum_{x=0}^{T+1} h(x)\left|\phi_{1}(x)\right|^{p}>0
$$

This contradicts $\lambda_{n}<\mu_{1} / f_{0}$.

\section{Directional turn of bifurcation}

In this section, we show that the connected components grow to the left at some point under (A4) condition.

In Lemma 3.3 and throughout, we use the following well-known conceptions of a generalized zero and a simple generalized zero at $t \in \mathbb{T}$ in [9].

Definition 3.1 Suppose that a function $y: \hat{\mathbb{T}} \rightarrow \mathbb{R}$. If $y\left(t_{0}\right)=0$, then $t_{0}$ is a zero of $y$. If $y\left(t_{0}\right)=0$ or $y\left(t_{0}\right) y\left(t_{0}+1\right)<0$ for some $t_{0} \in\{1, \ldots, T-1\}$, then $y$ has a generalized zero at $t_{0} \in \mathbb{T}$.

Lemma 3.2 Assume that (A1) holds. Let $u$ be a positive solution of (1.1). Then there exists $t_{0} \in \mathbb{T}$ such that $\|u\|=u\left(t_{0}\right)$. Moreover,

$$
\sigma\|u\| \leq u(x) \leq\|u\|, \quad x \in\left\{t \in \mathbb{Z}: \frac{T+1}{4} \leq t \leq \frac{3(T+1)}{4}\right\}=: I,
$$

where $\sigma=\min \left\{\frac{\min I}{T+1}, \frac{T+1-\max I}{T+1}\right\}$.

Proof It is an immediate consequence of the fact that $u$ is concave down in $\hat{\mathbb{T}}$.

Following similar arguments as in the proof of Lemma 3.1 of Dai and Ma [5], we have

Lemma 3.3 Let $P_{k} \geq p_{k}$ for $k \in[m, n+1]_{\mathbb{Z}}$. Also let $y(k), z(k)$ be solutions of the following difference equations:

$$
\begin{aligned}
& \Delta\left[\varphi_{p}(\Delta y(k))\right]+p_{k} \varphi_{p}(y(k+1))=0, \\
& \Delta\left[\varphi_{p}(\Delta z(k))\right]+P_{k} \varphi_{p}(z(k+1))=0,
\end{aligned}
$$


respectively. If $y(m)=y(n+1)=0$ but without any generalized zeros in $[m+1, n]_{\mathbb{Z}}$, then either there exists $\tau \in[m+1, n]_{\mathbb{Z}}$ such that $\tau$ is a generalized zero of $z$ or $P_{k}=p_{k}$ and $\frac{\Delta y(k)}{y(k)}=\frac{\Delta z(k)}{z(k)}$.

Proof If $z$ has a generalized zero in $[m+1, n]_{\mathbb{Z}}$, the conclusion is done. If there is no generalized zero of $z$ on $[m, n+1]_{\mathbb{Z}}$, then we can assume without loss of generality that $y>0$, $z>0$ in $[m+1, n]_{\mathbb{Z}}$. By the Picone-type identity $[11,20]$, we have

$$
\begin{aligned}
& \Delta\left\{\frac{y(k)}{\varphi_{p}(z(k))}\left[\varphi_{p}(z(k)) \varphi_{p}(\Delta y(k))-\varphi_{p}(y(k)) \varphi_{p}(\Delta z(k))\right]\right\} \\
& =\left(P_{k}-p_{k}\right)|y(k+1)|^{\alpha} \\
& \quad+\left\{|\Delta y(k)|^{\alpha}-\frac{\varphi_{p}(\Delta z(k))}{\varphi_{p}(z(k+1))}|y(k+1)|^{\alpha}+\frac{\varphi_{p}(\Delta z(k))}{\varphi_{p}(z(k))}|y(k)|^{\alpha}\right\} .
\end{aligned}
$$

By a direct computation, one has

$$
\begin{aligned}
& \frac{y(n+1)}{\varphi_{p}(z(n+1))}\left[\varphi_{p}(z(n+1)) \varphi_{p}(\Delta y(n+1))-\varphi_{p}(y(n+1)) \varphi_{p}(\Delta z(n+1))\right] \\
& \quad-\frac{y(m)}{\varphi_{p}(z(m))}\left[\varphi_{p}(z(m)) \varphi_{p}(\Delta y(m))-\varphi_{p}(y(m)) \varphi_{p}(\Delta z(m))\right] \\
& =\sum_{k=m}^{n}\left\{\left(P_{k}-p_{k}\right)|y(k+1)|^{\alpha}\right. \\
& \left.\quad+\left[|\Delta y(k)|^{\alpha}-\frac{\varphi_{p}(\Delta z(k))}{\varphi_{p}(z(k+1))}|y(k+1)|^{\alpha}+\frac{\varphi_{p}(\Delta z(k))}{\varphi_{p}(z(k))}|y(k)|^{\alpha}\right]\right\} .
\end{aligned}
$$

The left-hand side of (3.2) equals zero. Hence, the right-hand side of (3.2) also equals zero.

Since

$$
|\Delta y(k)|^{\alpha}-\frac{\varphi_{p}(\Delta z(k))}{\varphi_{p}(z(k+1))}|y(k+1)|^{\alpha}+\frac{\varphi_{p}(\Delta z(k))}{\varphi_{p}(z(k))}|y(k)|^{\alpha} \geq 0, \quad k \in[m, n]_{\mathbb{Z}}
$$

and the equality holds if and only if $\Delta y(k)=y(k)(\Delta z(k) / z(k))$, we conclude that there exists a constant $v \neq 0$ such that $z(k)=v y(k)$ and $P_{k}=p_{k}$.

Lemma 3.4 Assume that (A1) and (A4) hold. Let $u$ be a positive solution of (1.1) with $\|u\|=\frac{1}{\sigma} s_{0}$. Then $\lambda<\mu_{1} / f_{0}$.

Proof Let $u$ be a positive solution of (1.1). It follows from Lemma 3.2 that

$$
\sigma\|u\| \leq u(x) \leq\|u\|, \quad x \in I
$$

We note that $u$ is a solution of

$$
\Delta\left[\varphi_{p}(\Delta(u(x)))\right]+\lambda h(x) \frac{f(u(x))}{\varphi_{p}(u(x))} \varphi_{p}(u(x))=0, \quad x \in I .
$$


Suppose on the contrary that $\lambda \geq \mu_{1} / f_{0}$. Then, for $x \in I$, we have from (A4) that

$$
\lambda h(x) \frac{f(u(x))}{u(x)} \geq \frac{\mu_{1}}{f_{0}} h_{*} \frac{f_{0}}{\mu_{1} h_{*}} \chi_{1}=\chi_{1} .
$$

Choose $b>0$ such that $[b, b+\hat{t}]_{\mathbb{Z}} \subset I$. Set

$$
y(x)=w_{1}(x-b), \quad x \in[b, b+\hat{t}]_{\mathbb{Z}},
$$

then

$$
\left\{\begin{array}{l}
\Delta\left[\varphi_{p}(\Delta(y(x-1)))\right]+\chi_{1} \varphi_{p}(y(x))=0, \quad x \in[b+1, b+\hat{t}-1]_{\mathbb{Z}} \\
y(b)=0, \quad y(b+\hat{t})=0 .
\end{array}\right.
$$

It deduces from Lemma 3.3 that $u$ has at least one generalized zero on $I$. This contradicts the fact that $u(x)>0$ on $I$.

\section{Proof of Theorem 1.1}

The main ingredient of this section is a priori estimate, and finally we shall give a proof of Theorem 1.1.

Lemma 4.1 Assume that (A2) and (A3) hold. Let $u$ be a positive solution of (1.1). Then there exists $\lambda_{*}>0$ such that $\lambda \geq \lambda_{*}$.

Proof From Lemma 2.3, there exists a constant $C>0$, which is independent of $u$, such that (2.2) holds. Let $\|u\|=u\left(x_{0}\right)$, then it follows from (2.2) that

$$
\begin{aligned}
\|u\| & =u\left(x_{0}\right) \\
& =\sum_{s=0}^{x_{0}-1} \Delta(u(s)) \leq \sum_{s=0}^{x_{0}-1} \lambda^{\frac{1}{p-1}} C\|u\| \leq \lambda^{\frac{1}{p-1}} C T\|u\|,
\end{aligned}
$$

that is, $\lambda \geq(C T)^{1-p}$.

Lemma 4.2 Assume that (A1), (A2), and (A3) hold. Let $J$ be an interval in $(0,+\infty)$. Then there exists a constant $M_{J}>0$ such that, for all $\lambda \in J$, one has that all possible positive solutions $u$ of (1.1) satisfy $\|u\| \leq M_{J}$.

Proof Suppose on the contrary that there exists a sequence $\left\{u_{n}\right\}$ of positive solutions of (1.1) with $\left\{\lambda_{n}\right\} \subset J \triangleq[a, b]$ and $\left\|u_{n}\right\| \rightarrow \infty$ as $n \rightarrow \infty$. Let

$$
\beta \in\left(0, \frac{1}{b \varphi_{p}\left(\gamma_{p} Q T\right)}\right)
$$

where $\gamma_{p}=\max \left\{1, \frac{2-p}{2^{p-1}}\right\}, Q=\varphi_{p}^{-1}\left(\sum_{s=1}^{T} h(s)\right)$. Then, by (A3), there exists $u_{\beta}>0$ such that $u>u_{\beta}$ implies $f(u)<\beta u^{p-1}$. 
Let $m_{\beta} \triangleq \max _{u \in\left[0, u_{\beta}\right]} f(u)$ and let $A_{n} \triangleq\left\{t \in \mathbb{T}: u_{n}(t) \leq u_{\beta}\right\}$ and $B_{n} \triangleq\left\{t \in \mathbb{T}: u_{n}(t)>u_{\beta}\right\}$. Put $\Delta u_{n}\left(\delta_{n}\right)=0$ or $\Delta u_{n}\left(\delta_{n}-1\right) \Delta u_{n}\left(\delta_{n}\right)<0$. Then we have

$$
\begin{aligned}
\left\|u_{n}\left(\delta_{n}\right)\right\| & =\sum_{s=1}^{\delta_{n}} \varphi_{p}^{-1}\left(\varphi_{p}\left[\Delta u_{n}\left(\delta_{n}\right)\right]+\sum_{t=s}^{\delta_{n}} \lambda_{n} h(t) f\left(u_{n}(t)\right)\right) \\
& \leq \sum_{s=1}^{\delta_{n}} \varphi_{p}^{-1}\left(\sum_{t=1}^{\delta_{n}} \lambda_{n} h(t) f\left(u_{n}(t)\right)\right) \\
& \leq \varphi_{p}^{-1}\left(\lambda_{n}\right) \sum_{s=1}^{\delta_{n}} \varphi_{p}^{-1}\left(\sum_{t \in A_{n}} h(t) f\left(u_{n}(t)\right)+\sum_{t \in B_{n}} h(t) f\left(u_{n}(t)\right)\right)
\end{aligned}
$$

for $0 \leq s \leq \delta_{n}$. Thus

$$
\frac{1}{\varphi_{p}^{-1}\left(\lambda_{n}\right)} \leq \gamma_{p} \sum_{s=1}^{\delta_{n}}\left[\frac{\varphi_{p}^{-1}\left(m_{\beta}\right) Q}{\left\|u_{n}\right\|}+\varphi_{p}^{-1}\left(\sum_{t \in B_{n}} \frac{h(t) f\left(u_{n}(t)\right)}{\left\|u_{n}\right\|^{p-1}}\right)\right] .
$$

On $B_{n}, u_{n}(s)>u_{\beta}$ implies $\frac{f\left(u_{n}(s)\right)}{\left\|u_{n}\right\|^{p-1}} \leq \frac{f\left(u_{n}(s)\right)}{u_{n}^{p-1}(s)} \leq \beta$. And accordingly

$$
\frac{1}{\varphi_{p}^{-1}\left(\lambda_{n}\right)} \leq \gamma_{p} T\left[\frac{\varphi_{p}^{-1}\left(m_{\beta}\right) Q}{\left\|u_{n}\right\|}+\varphi_{p}^{-1}(\beta) Q\right] .
$$

Since $0<a<\lambda_{n} \leq b$ for all $n$, we have $\frac{1}{\varphi_{p}^{-1}\left(\lambda_{n}\right)} \geq \frac{1}{\varphi_{p}^{-1}(b)}$ for all $n$, and

$$
\frac{1}{\varphi_{p}^{-1}(b)} \leq \gamma_{p} T\left[\frac{\varphi_{p}^{-1}\left(m_{\beta}\right) Q}{\left\|u_{n}\right\|}+\varphi_{p}^{-1}(\beta) Q\right]
$$

According to the fact $\left\|u_{n}\right\| \rightarrow \infty$ as $n \rightarrow \infty$, we get

$$
\frac{1}{\varphi_{p}^{-1}(b)} \leq \gamma_{p} T \varphi_{p}^{-1}(\beta) Q<\gamma_{p} T \varphi_{p}^{-1}\left(\frac{1}{b \varphi_{p}\left(\gamma_{p} Q T\right)}\right) Q<\frac{1}{\varphi_{p}^{-1}(b)} .
$$

This contradiction completes the proof.

Lemma 4.3 Assume that (A1), (A2), and (A3) hold. Let $u$ be a positive solution of (1.1). Then there exists a constant $C>0$ independent of $u$ such that $\lambda f_{-}(\|u\|) \leq C$, where $f_{-}(s)=$ $\min _{\sigma s \leq t \leq s} \frac{f(t)}{t}$.

Proof It is well known that

$$
\left\{\begin{array}{l}
\Delta\left[\varphi_{p}(\Delta u(x-1))\right]+\lambda h(x) f(u(x))=0, \quad x \in \mathbb{T} \\
u(0)=u(T+1)=0
\end{array}\right.
$$

is equivalent to the operator equation

$$
u=T(u),
$$


where

$$
T(u)(x)= \begin{cases}\sum_{s=0}^{x-1} \varphi_{q}\left(\sum_{\tau=s+1}^{\sigma_{u}} \lambda h(\tau) f(u(\tau))\right), & x \in\left[1, \sigma_{u}\right]_{\mathbb{Z}}, \\ \sum_{s=x}^{T} \varphi_{q}\left(\sum_{\tau=s+1}^{\sigma_{u}} \lambda h(\tau) f(u(\tau))\right), & x \in\left[\sigma_{u}, T\right]_{\mathbb{Z}},\end{cases}
$$

with $\frac{1}{p}+\frac{1}{q}=1, \sigma_{u} \in \mathbb{T}$ is the unique solution of

$$
c_{u}=\sum_{\tau=1}^{\sigma_{u}} \lambda h(\tau) f(u(\tau))
$$

and $c_{u}$ is the unique solution of

$$
\sum_{s=0}^{T}\left(c_{u}-\sum_{\tau=1}^{s} \lambda h(\tau) f(u(\tau))\right)=0 .
$$

Let $\hat{t} \in \mathbb{T}$ be as in (1.3) and

$$
J_{1}:=[\min I, \hat{t}], \quad J_{2}:=[\hat{t}, \max I] .
$$

Then we have

$$
\begin{aligned}
& \|u\| \geq|u(\hat{t})| \\
& = \begin{cases}\sum_{s=0}^{\hat{t}-1} \varphi_{q}\left(\sum_{\tau=s+1}^{\sigma_{u}} \lambda h(\tau) f(u(\tau)),\right. & \hat{t} \in\left[1, \sigma_{u}\right]_{\mathbb{Z}}, \\
\sum_{s=\hat{t}}^{T} \varphi_{q}\left(\sum_{\tau=\sigma_{u}}^{s} \lambda h(\tau) f(u(\tau)),\right. & \hat{t} \in\left[\sigma_{u}, T\right]_{\mathbb{Z}},\end{cases} \\
& \geq \begin{cases}\sum_{s \in J_{1}} \varphi_{q}\left(\sum_{\tau=s+1}^{\sigma_{u}} \lambda h(\tau) f(u(\tau)),\right. & \hat{t} \in\left[1, \sigma_{u}\right]_{\mathbb{Z}}, \\
\sum_{s \in J_{2}} \varphi_{q}\left(\sum_{\tau=\sigma_{u}}^{s} \lambda h(\tau) f(u(\tau)),\right. & \hat{t} \in\left[\sigma_{u}, T\right]_{\mathbb{Z}},\end{cases} \\
& = \begin{cases}\|u\| \sum_{s \in J_{1}} \varphi_{q}\left(\sum_{\tau=s+1}^{\sigma_{u}} \lambda h(\tau) \frac{f(u(\tau))}{\varphi_{p}(u(\tau))} \frac{\varphi_{p}(u(\tau))}{\|u\|^{p-1}}\right), & \hat{t} \in\left[1, \sigma_{u}\right]_{\mathbb{Z}}, \\
\|u\| \sum_{s \in J_{2}} \varphi_{q}\left(\sum_{\tau=\sigma_{u}}^{s} \lambda h(\tau) \frac{f(u(\tau))}{\varphi_{p}(u(\tau))} \frac{\varphi_{p}(u(\tau))}{\|u\|^{p-1}}\right), & \hat{t} \in\left[\sigma_{u}, T\right]_{\mathbb{Z}},\end{cases} \\
& \geq \begin{cases}\|u\| \sum_{s \in J_{1}} \varphi_{q}\left(\sum_{\tau=s+1}^{\sigma_{u}} \lambda h(\tau) \min _{\tau \in J_{1}} \frac{f(u(\tau))}{\varphi_{p}(u(\tau))} \frac{\varphi_{p}(u(\tau))}{\|u\|^{p-1}}\right), & \hat{t} \in\left[1, \sigma_{u}\right]_{\mathbb{Z}}, \\
\|u\| \sum_{s \in J_{2}} \varphi_{q}\left(\sum_{\tau=\sigma_{u}}^{s} \lambda h(\tau) \min _{\tau \in J_{2}} \frac{f(u(\tau))}{\varphi_{p}(u(\tau))} \frac{\varphi_{p}(u(\tau))}{\|u\|^{p-1}}\right), & \hat{t} \in\left[\sigma_{u}, T\right]_{\mathbb{Z}},\end{cases} \\
& \geq \begin{cases}\|u\| \sum_{s \in J_{1}} \varphi_{q}\left(\sum_{\tau=s+1}^{\sigma_{u}} \lambda h(\tau) \min _{\sigma\|u\| \leq r \leq\|u\|} \frac{f(r)}{\varphi_{p}(r)} \frac{\varphi_{p}(u(\tau))}{\|u\| \|^{p-1}}\right), & \hat{t} \in\left[1, \sigma_{u}\right]_{\mathbb{Z}}, \\
\|u\| \sum_{s \in J_{2}} \varphi_{q}\left(\sum_{\tau=\sigma_{u}}^{s} \lambda h(\tau) \min _{\sigma\|u\| \leq r \leq\|u\|} \frac{f(r)}{\varphi_{p}(r)} \frac{\varphi_{p}(u(\tau))}{\|u\|^{p-1}}\right), & \hat{t} \in\left[\sigma_{u}, T\right]_{\mathbb{Z}},\end{cases} \\
& \geq \begin{cases}\|u\| \sum_{s \in J_{1}} \varphi_{q}\left(\sum_{\tau=S+1}^{\sigma_{u}} \lambda h(\tau) f(\|u\|) \frac{\varphi_{p}(u(\tau))}{\|u\|^{p-1}}\right), & \hat{t} \in\left[1, \sigma_{u}\right]_{\mathbb{Z}}, \\
\|u\| \sum_{s \in J_{2}} \varphi_{q}\left(\sum_{\tau=\sigma_{u}}^{s} \lambda h(\tau)\right]_{-}(\|u\|) \frac{\varphi_{p}(u(\tau))}{\left.\|u\|^{\mid p^{-1}}\right),} & \hat{t} \in\left[\sigma_{u}, T\right]_{\mathbb{Z}},\end{cases} \\
& \geq \begin{cases}\|u\| \sum_{s \in J_{1}} \varphi_{q}\left(\sigma_{u}-s-1\right)\left[\lambda h_{*} f(\|u\|) \sigma^{p-1}\right]^{q-1}, & \hat{t} \in\left[1, \sigma_{u}\right]_{\mathbb{Z}}, \\
\|u\| \sum_{s \in J_{2}} \varphi_{q}\left(s+1-\sigma_{u}\right)\left[\lambda h_{*}-(\|u\|) \sigma^{p-1}\right]^{q-1}, & \hat{t} \in\left[\sigma_{u}, T\right]_{\mathbb{Z}},\end{cases} \\
& \geq \begin{cases}\|u\| \sum_{s \in J_{1}} \varphi_{q}(\hat{t}-s-1)\left[\lambda h_{*} f(\|u\|) \sigma^{p-1}\right]^{q-1}, & \hat{t} \in\left[1, \sigma_{u}\right]_{\mathbb{Z}}, \\
\|u\| \sum_{s \in J_{2}} \varphi_{q}(s+1-\hat{t})\left[\lambda h_{*}-\bar{f}(\|u\|) \sigma^{p-1}\right]^{q-1}, & \hat{t} \in\left[\sigma_{u}, T\right]_{\mathbb{Z}},\end{cases}
\end{aligned}
$$




$$
\begin{aligned}
& \geq\|u\| \min \left\{\sum_{s \in J_{1}} \varphi_{q}(\hat{t}-s-1), \sum_{s \in J_{2}} \varphi_{q}(s+1-\hat{t})\right\}\left[\lambda h_{*} f(\|u\|) \sigma^{p-1}\right]^{q-1} \\
& \geq \sigma\|u\| \min \left\{\sum_{s \in J_{1}} \varphi_{q}(\hat{t}-s-1), \sum_{s \in J_{2}} \varphi_{q}(s+1-\hat{t})\right\}\left[\lambda h_{*} f(\|u\|)\right]^{q-1} .
\end{aligned}
$$

This completes the proof.

Lemma 4.4 Assume that (A1), (A2), (A3), and (A4) hold. Then there exists $\left\{\left(\lambda_{n}, u_{n}\right)\right\}$ such that $\left(\lambda_{n}, u_{n}\right) \in \mathcal{C}, \lambda_{n} \rightarrow \infty$ and $\left\|u_{n}\right\| \rightarrow \infty$ as $n \rightarrow \infty$.

Proof It follows from Lemma 2.2 that $\mathcal{C}$ is unbounded, so there exists a sequence $\left\{\left(\lambda_{n}, u_{n}\right)\right\}$ of solutions of (1.1) such that $\left(\lambda_{n}, u_{n}\right) \in \mathcal{C}$ and $\left|\lambda_{n}\right|+\left\|u_{n}\right\| \rightarrow \infty$. Moreover, Lemma 4.1 implies that $\lambda_{n}>0$. Suppose on the contrary that there exists a bounded subsequence $\left\{\left(\lambda_{n_{k}}, u_{n_{k}}\right)\right\}$. Then, from Lemma 4.2, $\left\|u_{n_{k}}\right\|$ is bounded, which contradicts the fact that $\left|\lambda_{n}\right|+$ $\left\|u_{n}\right\| \rightarrow \infty$. Therefore, $\lambda_{n} \rightarrow \infty$. Then Lemma 4.3 implies that $f\left(\left\|u_{n}\right\|\right) \rightarrow 0$. It deduces from (A4) that $\left\|u_{n}\right\| \rightarrow \infty$.

Proof of Theorem 1.1 Let $\mathcal{C}$ be as in Lemma 2.2 .

It follows from Lemma 2.5 that $\mathcal{C}$ is emanating from $\left(\mu_{1} / f_{0}, 0\right)$ and goes rightward. Let $\left\{\left(\lambda_{n}, u_{n}\right)\right\}$ be as in Lemma 4.4. Then there exists $\left(\lambda_{0}, u_{0}\right) \in \mathcal{C}$ such that $\left\|u_{0}\right\|=\frac{1}{\sigma} s_{0}$. By Lemma 3.4, one has $\lambda_{0}<\mu_{1} / f_{0}$.

By virtue of Lemmas $2.5,3.4$, and $4.2, \mathcal{C}$ passes through some points $\left(\mu_{1} / f_{0}, v_{1}\right)$ and $\left(\mu_{1} / f_{0}, v_{2}\right)$ with

$$
\left\|v_{1}\right\|<\frac{1}{\sigma} s_{0}<\left\|v_{2}\right\|
$$

By Lemmas $2.5,3.4$, and 4.2 again, there exist $\underline{\lambda}$ and $\bar{\lambda}$ which satisfy $0<\underline{\lambda}<\mu_{1} / f_{0}<\bar{\lambda}$ and

(i) if $\lambda \in\left(\mu_{1} / f_{0}, \bar{\lambda}\right]$, then there exist $u$ and $v$ such that $(\lambda, u),(\lambda, v) \in \mathcal{C}$ and

$$
\|u\|<\|v\|<\frac{1}{\sigma} s_{0}
$$

(ii) if $\lambda \in\left[\underline{\lambda}, \mu_{1} / f_{0}\right]$, then there exist $u$ and $v$ such that $(\lambda, u),(\lambda, v) \in \mathcal{C}$ and

$$
\|u\|<\frac{1}{\sigma} s_{0}<\|v\| .
$$

Define

$$
\lambda^{*}=\sup \{\bar{\lambda}: \bar{\lambda} \text { satisfies (i) }\}, \quad \lambda_{*}=\inf \{\underline{\lambda}: \underline{\lambda} \text { satisfies (ii) }\} .
$$

Then, by the standard argument, (1.1) has a positive solution at $\lambda=\lambda_{*}$ and $\lambda=\lambda^{*}$, respectively. Since $\mathcal{C}$ passes through $\left(\mu_{1} / f_{0}, \nu_{2}\right)$ and $\left(\lambda_{n}, u_{n}\right)$, Lemma 3.4 implies that there exists $w$ such that, for each $\lambda>\mu_{1} / f_{0}$, one has $(\lambda, w) \in \mathcal{C}$ and $\|w\|>\frac{1}{\sigma} s_{0}$. This completes the proof. $\square$ 
Funding

This work was supported by the National Natural Science Foundation of China (No. 11671322).

\section{Abbreviations}

Not applicable.

Availability of data and materials

Data sharing not applicable to this article as no datasets were generated.

Competing interests

All of the authors of this article claim that together they have no competing interests.

\section{Authors' contributions}

The authors claim that the research was realized in collaboration with the same responsibility. All authors read and approved the last of the manuscript.

\section{Publisher's Note}

Springer Nature remains neutral with regard to jurisdictional claims in published maps and institutional affiliations.

Received: 18 October 2019 Accepted: 28 April 2020 Published online: 11 May 2020

\section{References}

1. Agarwal, R.P., Perera, K., O'Regan, D.: Multiple positive solutions of singular discrete $p$-Laplacian problems via variational methods. Adv. Differ. Equ. 2005, Article ID 690272 (2005)

2. Ambrosio, V., Molica Bisci, G., Repovš, D.: Nonlinear equations involving the square root of the Laplacian. Discrete Contin. Dyn. Syst., Ser. S 12(2), 401-411 (2019)

3. Bai, D., Chen, Y.: Global continuum of positive solutions for discrete $p$-Laplacian eigenvalue problems. Appl. Math. 60(4), 343-353 (2015)

4. Chu, J., Jiang, D.: Eigenvalues and discrete boundary value problems for the one-dimensional p-Laplacian. J. Math. Anal. Appl. 305(2), 452-465 (2005)

5. Dai, G., Ma, R.: Unilateral global bifurcation for $p$-Laplacian with non- $p$ - 1-linearization nonlinearity. Discrete Contin. Dyn. Syst. 35(1), 99-116 (2015)

6. Feng, T., Yu, M.: Nonlinear Picone identities to pseudo $p$-Laplace operator and applications. Bull. Iran. Math. Soc. 43, 2517-2530 (2017)

7. He, Z:: On the existence of positive solutions of $p$-Laplacian difference equations. J. Comput. Appl. Math. 161, 193-201 (2003)

8. Jiang, L., Zhou, Z.: Three solutions to Dirichlet boundary value problems for $p$-Laplacian difference equations. Adv. Differ. Equ. 2008, Article ID 345916 (2008)

9. Kelley, W.G., Peterson, A.C.: Difference Equations: An Introduction with Applications. Academic Press, New York (1991)

10. Li, Y., Lu, L.: Existence of positive solutions of $p$-Laplacian difference equations. Appl. Math. Lett. 19, 1019-1023 (2006)

11. Mař́k, R.: Comparison theorems for half-linear second order difference equations. Arch. Math. 59(7), 513-518 (2000)

12. Molica Bisci, G., Repovš, D.: Existence of solutions for $p$-Laplacian discrete equations. Appl. Math. Comput. 242, 454-461 (2014)

13. Molica Bisci, G., Repovš, D., Vilasi, L.: Multiple solutions of nonlinear equations involving the square root of the Laplacian. Appl. Anal. 96(9), 1483-1496 (2017)

14. Nastasi, A., Vetro, C.: A note on homoclinic solutions of $(p, q)$-Laplacian difference equations. J. Differ. Equ. Appl. 25(3), 331-341 (2019)

15. Nastasi, A., Vetro, C., Vetro, F.: Positive solutions of discrete boundary value problems with the $(p, q)$-Laplacian operator. Electron. J. Differ. Equ. 2017, Article ID 225 (2017)

16. Papageorgiou, N.S., Rǎdulescu, V.D., Repovš, D.D.: Pairs of positive solutions for resonant singular equations with the p-Laplacian. Electron. J. Differ. Equ. 2017, Article ID 249 (2017)

17. Papageorgiou, N.S., Rǎdulescu, V.D., Repovš, D.D.: Nonlinear Analysis—Theory and Methods. Springer Monographs in Mathematics. Springer, Cham (2019)

18. Papageorgiou, N.S., Rǎdulescu, V.D., Repovš, D.D.: Nonlinear nonhomogeneous boundary value problems with competition phenomena. Appl. Math. Optim. 80(1), 251-298 (2019)

19. Picone, M.: Sui valori eccezionali di un parametro da cui dipende un'equazione differenziale lineare ordinaria del second'ordine. Ann. Sc. Norm. Super. Pisa 11, 1-141 (1909)

20. Řehák, P.: Oscillatory properties of second order half-linear difference equations. Czechoslov. Math. J. 51, 303-321 (2001)

21. Sim, I., Tanaka, S.: Three positive solutions for one-dimensional $p$-Laplacian problem with sign-changing weight. Appl. Math. Lett. 49, 42-50 (2015)

22. Yoshida, N.: Oscillation criteria for half-linear elliptic inequalities with $p(x)$-Laplacians via Riccati method. Nonlinear Anal. 74, 2563-2575 (2011) 